\title{
Performance Evaluation of Moving Small-Cell Network with Proactive Cache
}

\author{
Young Min Kwon, ${ }^{1}$ Syed Tariq Shah, ${ }^{1}$ JaeSheung Shin, ${ }^{2}$ \\ Ae-Soon Park, ${ }^{2}$ and Min Young Chung ${ }^{1}$ \\ ${ }^{1}$ College of Information and Communication Engineering, Sungkyunkwan University, 2066 Seobu-Ro, Jangan-Gu, \\ Suwon, Gyeonggi-Do 16419, Republic of Korea \\ ${ }^{2}$ Mobile Access Research Division, Electronics and Telecommunications Research Institute, 138 Gajeongno, Yuseong-gu, \\ Daejeon 34129, Republic of Korea \\ Correspondence should be addressed to Min Young Chung; mychung@skku.edu
}

Received 21 January 2016; Revised 20 June 2016; Accepted 21 June 2016

Academic Editor: Juan C. Cano

Copyright ( 2016 Young Min Kwon et al. This is an open access article distributed under the Creative Commons Attribution License, which permits unrestricted use, distribution, and reproduction in any medium, provided the original work is properly cited.

\begin{abstract}
Due to rapid growth in mobile traffic, mobile network operators (MNOs) are considering the deployment of moving small-cells (mSCs). $\mathrm{mSC}$ is a user-centric network which provides voice and data services during mobility. $\mathrm{mSC}$ can receive and forward data traffic via wireless backhaul and sidehaul links. In addition, due to the predictive nature of users demand, mSCs can proactively cache the predicted contents in off-peak-traffic periods. Due to these characteristics, MNOs consider mSCs as a cost-efficient solution to not only enhance the system capacity but also provide guaranteed quality of service (QoS) requirements to moving user equipment (UE) in peak-traffic periods. In this paper, we conduct extensive system level simulations to analyze the performance of mSCs with varying cache size and content popularity and their effect on wireless backhaul load. The performance evaluation confirms that the QoS of moving small-cell UE (mSUE) notably improves by using mSCs together with proactive caching. We also show that the effective use of proactive cache significantly reduces the wireless backhaul load and increases the overall network capacity.
\end{abstract}

\section{Introduction}

Due to the increasing number of smart phone devices and data services, the users demand for mobile data traffic has also increased. Global mobile traffic will nearly increase tenfold until 2018 [1]. To accommodate this emerging demand of data traffic, mobile network operators (MNOs) have already adopted advanced communication techniques such as orthogonal frequency division multiple access (OFDMA), multiple input multiple output (MIMO), and carrier aggregation (CA). It is possible to make the spectrum efficiency reach its theoretical limit in $4 \mathrm{G}$ mobile network by using these technologies. However, the networks only implementing these advanced radio access and transmission technologies will not be able to accommodate the tremendous increment of mobile traffic and it may exhaust the available system capacity of $4 \mathrm{G}$ mobile networks. Thus, MNOs have considered heterogeneous networks (HetNets) in order to continuously improve the systems capacity by adding more base stations $[2,3]$.

The HetNet terminology indicates that various types of fixed small-cells (fSCs) such as pico- and femtocell coexist in a macrocell. fSCs can share the traffic overload of macrocell by providing mobile services to densely populated areas such as hotspots [4]. However, fSCs using wired backhaul have drawback in terms of signaling overheard, infrastructure cost, and mobility [5]. When many fSCs densely exist in cellular networks, frequent handovers occur between macrocell and fSCs [6]. For successful handover, both base stations of macrocell and fSCs should exchange control messages via wired backhaul comprised of several network entities [7]. Thus, dense deployment of fSCs increases signaling load in the wired backhaul. Secondly, existing fSCs require wired backhauls such as optical fiber or coaxial cable, in order to connect them to the core network. Laying these wired 
backhaul is not a very cost-effective solution for MNOs. Moreover, fSCs using a wired backhaul cannot consistently provide wireless broadband services to users that ride public transportation vehicles [8]. Recently, working group (WG) of $3 \mathrm{GPP}$ standardization has investigated the moving cell utilizing the wireless backhaul as a solution to overcome the limitations of fSCs [9].

In this paper, we introduce the concept of moving smallcell (mSC) with various transmission paths, that is, wireless backhaul, sidehaul, and caching transmission. mSCs are usercentric networks that autonomously establish connections between users and provide the voice and data services while moving [10]. mSCs communicate with their respective MBSs via wireless backhaul links. mSCs can also exchange data through wireless sidehaul links among neighboring mSCs. Due to predictable nature of users, the nodes in the network track can learn and construct the users' demand profiles in order to predict their future requests effectively. Thus, in the proposed mSC network, each $\mathrm{mSC}$ has a storage capability to cache the predicted contents. The proposed caching mechanism is proactive in principle and it aims to anticipate users demands. It can reduce the backhaul load by saving the scarce frequency resources. Due to these unique characteristics, $\mathrm{mSC}$ has several advantages over other fSCs. By supporting group handover, mSCs can reduce both signaling overhead and handover failure probability [11]. Since wireless backhaul and sidehaul links do not require any additional deployment cost, mSCs can become a cost-efficient solution to enhance the systems capacity [12]. Furthermore, the proposed proactive caching mechanism used in mSCs can not only reduce the traffic load of wireless backhaul link but also guarantee quality of service (QoS) performance in peak-traffic hours $[13,14]$.

The deployment of mSCs can enhance system capacity and accommodate the increasing mobile traffic with reasonable cost. Instead of deploying new fSCs, mSCs can be utilized as a cost-effective solution to solve the temporary hotspot issues. Although mSCs have many advantages in terms of traffic distribution and system capacity, their performance is limited due to cotier interference among neighboring mSCs. Since mSCs accommodate all the data traffic of wireless backhaul link, wireless sidehaul link, and proactive content cache, it is obvious that the performance of $\mathrm{mSC}$ is affected by ratio between data traffic delivered via these various links. Thus, we have developed and conducted extensive system level simulations to analyze the effect of mSCs with proactive caching enabled in a multitier HetNet environment.

Contributions. System level simulation is one of the most useful methodologies to analyze the performance of various network scenarios [15]. A preliminary version of this paper appears in the 8th ACM International Conference on Ubiquitous Information Management and Communication (IMCOM), 2014 [16]. In this study, we first highlight the challenges associated with mSCs deployment in multitier HetNets scenarios. Then, in order to exploit the advantages of mSCs and proactive caching, we evaluate and compare the performance of mSCs in different multitier HetNet scenarios. We show the relation between contents popularity, cache size, and operating modes and their positive effects on overall network performance.

The rest of the paper is organized as follows. Section 2 presents the previous studies related to $\mathrm{mSCs}$ and proactive caching. In Section 3, we introduce the proposed $\mathrm{mSC}$ network, its architecture, and proactive caching mechanism used. Section 4 contains the detailed performance evaluation of proposed mSC network and Section 5 provides the conclusion of this paper.

\section{Related Works}

Due to unprecedented growth in mobile data traffic, network densification and modification in its current architecture are inevitable. In order to maximize the reuse of available frequency spectrum, introducing HetNets is one of the key solutions. HetNets can accommodate the growing demand of data traffic by deploying more small-cells in a given area $[2,17,18]$. In [19], Dhillon and others have proposed a tractable model for a K-tiers downlink HetNet. It shows that in an ideal HetNet scenario, beside severe interference, the network densification can still significantly enhance the overall network capacity. In order to provide better and reliable network services to moving users, the use of mSCs has been proposed, studied, and evaluated in [20-24].

The authors in [20] have shown that, in a coverage limited scenario the use of coordinated and cooperative relays in public vehicles can significantly improve the network experience of on-board moving users. In [8, 21-23], Sui and others have studied performance of moving relay node $(\mathrm{MRN})$, which is a type of mSCs, in cellular networks. MRNs are deployed in public transportation vehicles such as trains, trams, and buses in order to provide wireless broadband services to moving UE. Since MRN uses wireless backhaul link to connect to MBS, it can reduce the cost of wired backhaul link. In addition, by supporting group handover of all on-board UE, MRN can significantly reduce the signaling overhead and probability of handover failure. Compared to MBS, MRN is very close to its UE; therefore it can enhance the signal quality of the respective UE in access link. However, the performance of MRN mainly depends on the capacity of wireless backhaul link $[21,22]$. Since the capacity of wireless backhaul link is normally limited, it is difficult to increase the overall network capacity by deploying large number of MRNs significantly.

The ability to predict user demands and recent developments in context awareness and data storage has enabled the future networks to proactively cache the popular contents in advance [25-28]. The proactive caching technique in small-cells will not only reduce the backhaul load but also guarantee the QoS requirements in peak-traffic periods. In [25], Tadrous and others have studied the concept of proactive resource allocation by utilizing the predictability of user behavior for load balancing. Authors in [26] have proposed the idea of femtocaching in fSCs with very limited backhaul bandwidth and large storage capacity. Authors in [27] have studied the asymptotic scaling laws of caching in D2D communications. In their proposed distributed caching scheme, users store the popular contents and forward them to 


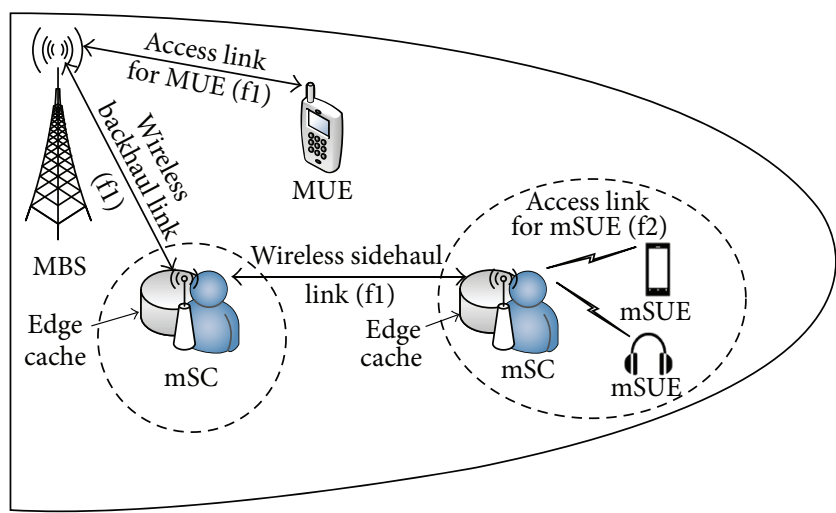

FIgURE 1: Moving small-cell network.

other users by using D2D communications. Bastug and others in [28] have examined two cases of proactive caching. First, in order to reduce the backhaul load, they have proposed a mechanism, which proactively caches the popular files in off-peak hours (e.g., at night) proactively. In second case, based on the social structure of the network, the proposed scheme predicts the set of potential users who can proactively cache and distribute the popular contents utilizing D2D communications.

Nonetheless, these studies on proactive caching have only considered the fSCs (pico- and femtocells) which usually have wired backhauls and do not have any backhaul bandwidth constraint. Moreover, they also rarely consider the mobility of either small-cells (picocells and femtocells) or users (D2D). These key aspects are the motivation behind this paper and the aim of this is to study the role of proactive caching in mSCs.

\section{Proposed Moving Small-Cell Network with Proactive Cache}

3.1. Network Architecture. The proposed mSC network consists of four network entities, MBS, macrocell UE (MUE), mSC, and moving small-cell UE (mSUE) as shown in Figure 1. MBS in mSC network provides wireless access link and backhaul link connections to its serving MUE and mSCs, respectively. Each $\mathrm{mSC}$ is a moving small-cell, which provides wireless broadband services to its serving mSUE in access links. To communicate between mSCs directly, mSCs can also establish wireless sidehaul connections with their neighboring mSCs. Based on measurement information, the MBS is also responsible for radio resource management of both wireless backhaul and sidehaul links of mSCs. Furthermore, in our proposed mSC network, each mSC has the ability to cache popular contents. If mSUE requests contents that are already stored in the cache of its connected $\mathrm{mSC}$, the $\mathrm{mSC}$ directly sends the contents to its mSUE. More detail on proactive caching is given in the next section.

As discussed earlier, due to wireless backhaul and sidehaul connectivity, mPCs can be deployed on moving vehicles to provide enhanced network services to moving UE. It is obvious that, instead of deploying large number of fSC,
$\mathrm{mSCs}$ are the cost-efficient technique to serve moving UE and increase the overall network capacity. In order to avoid severe interference between MUE and mSUE, both MBSs and $\mathrm{mSCs}$ in the proposed scheme use different frequency bands of $2.0 \mathrm{GHz}$ and $3.5 \mathrm{GHz}$ in their access links, respectively. Figure 2 shows the proposed channels and frequencies assignment scheme for wireless backhaul/sidehaul and access links of mSCs, MUE, and mSUE, respectively. In mSC network, in-band full duplex transmission may be used for wireless backhaul link. Thus, for wireless backhaul transmissions, mSCs share the same radio resources of uplink and downlink in $2 \mathrm{GHz}$ frequency band with MUE. Furthermore, mSCs also perform in-band half-duplex transmission for wireless sidehaul links, where they reuse the uplink radio resources of mSC backhaul and MUE in $2 \mathrm{GHz}$ frequency band. Unlike MUE, mSUE is very close to the serving mSCs; thus the transmit power of $\mathrm{mSC}$ is relatively lower than MBS.

\subsection{Proposed Proactive Caching Scheme for mSC Network.}

It is mentioned earlier in this paper that preloading and proactive caching can significantly reduce the traffic load on wireless backhaul link and conserve the scarce radio resources. The key issues of proactive caching are methods to decide caching data and an efficient mechanism to transmit the selected data (preloading) [29]. This paper focuses on the second key issue of cache preloading. We assume that, based on collaborative filtering (CF) tools [30], the MBS can effectively decide the popularity of the contents such as video contents (e.g., TV series and advertisements), web contents (e.g., daily news, blogs, and digests), and software update files (e.g., software drivers and patches) [31]. These contents are usually time-insensitive and available long before their scheduled publishing time. The effect on time-sensitive contents has not been evaluated in this paper; it is because we assume that MBSs transmit the selected contents to their respective mSCs in off-peak period (e.g., night time). In other words, the cache of mSCs in our proposed scheme is only updated in low traffic hours when the traffic load on backhaul link is very low [28]. In order to continuously update the cache with time-sensitive popular contents, a fulltime dedicated backhaul link is required. However, due to scarce availability of the radio resources, it is not feasible to fully dedicate certain backhaul resources only for cache management.

In order to make the preloading scheme more efficient, the MBS transmits the popular contents to mSCs in two possible modes: broadcasting and multicasting. If the content files are equally popular among all $\mathrm{mSCs}$ in the network, the MBS will broadcast the selected contents to all mSCs in the network. Similarly, if different content files are popular among different mSCs, the MBS will make groups of mSCs with same interest and it will multicast the desired contents to each particular group. Furthermore, in multicast mode mSCs of one group can exchange their cache contents with nearest neighboring $\mathrm{mSC}$ of other groups via sidehaul link. In other words, if the requested contents are available in neighboring $\mathrm{mSCs}$, the MBS will provide the necessary information (mSC ID, radio resources for sidehaul, and so on) of that particular $\mathrm{mSC}$ in order to establish sidehaul link. In our proposed 


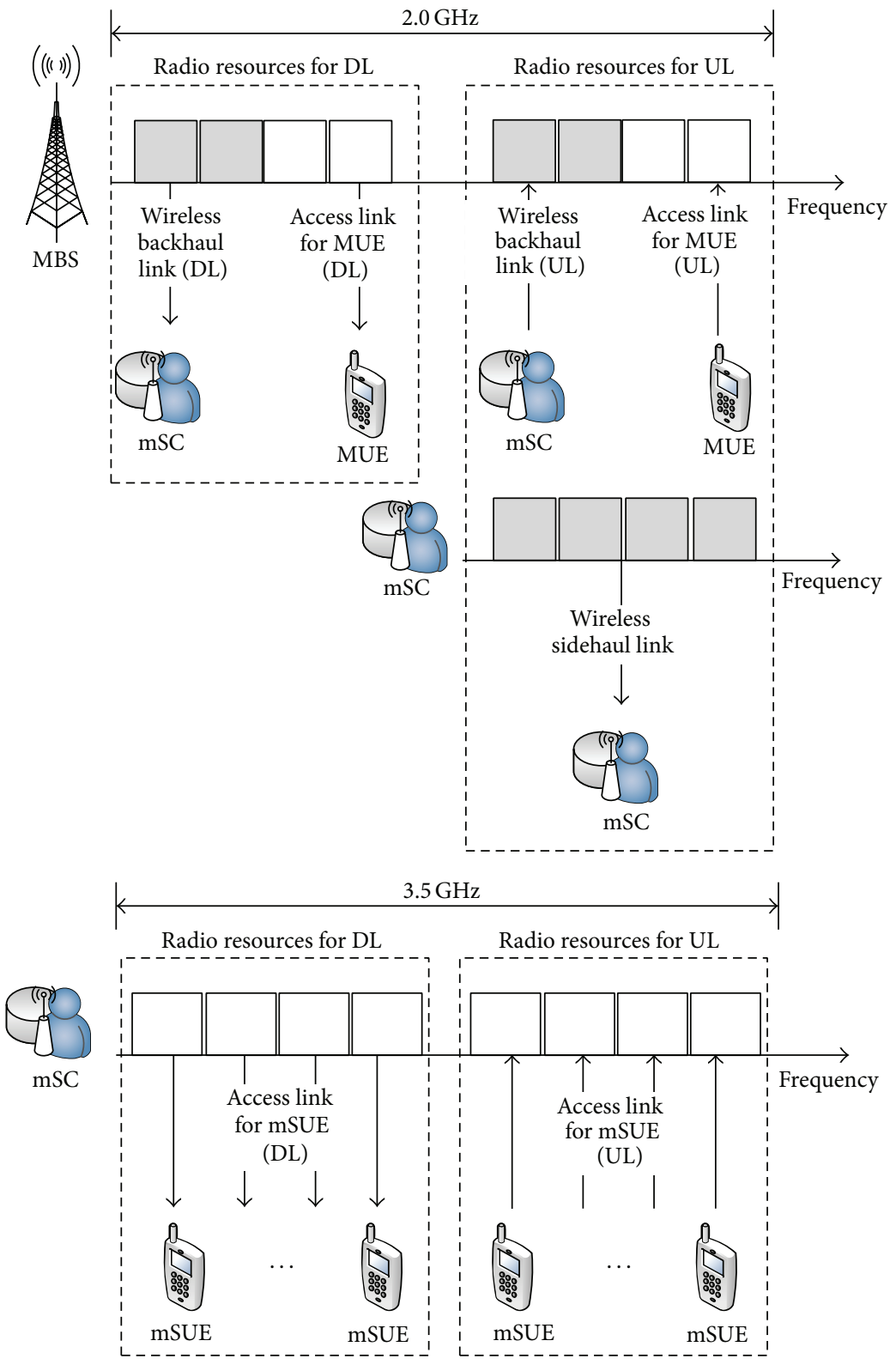

FIGURE 2: Channel assignment in $\mathrm{mSC}$ network.

network, mSCs can only establish sidehaul connection with neighboring mSCs that are located in the radius of 200 meters. Since, sidehaul links reuse the uplink frequencies of both mSCs and MUE, they can significantly reduce the backhaul traffic load. Note that the aim of our proposed scheme is to evaluate the performance of a fully loaded MSC network with active sidehaul links and proactive cache, under the constraint of limited wireless backhaul capacity. Therefore, in our proposed network model, we have considered that the number of mSCs in each macrocell and the number of mSUE pieces in each mSC are uniform and fixed. The aim of such network model is to find the upper bound of network capacity. Consequently, due to these considerations the traffic conditions of an mSC in our proposed network do not vary over time and the resource allocation is static. Figure 3 shows the proposed preloading scheme, where, during off-peak period, the backhaul bandwidth is divided into two parts, one for reactive backhaul traffic and the second for proactive broadcast/multicast caching traffic.

In our proposed mSC network, the network performance depends on three different factors: content popularity distribution, cache size of $\mathrm{mSC}$, and the number of multicasting groups. In this paper, popularity distributions are obtained from $\mathrm{ZipF}(\alpha)$ distribution [32]. It has been shown in [33, 34 ] that the global content popularity usually follows the $\mathrm{ZipF}$ distribution. It is also shown in [34] that a simple model for an independent request stream following a ZipF distribution is sufficient to capture certain asymptotic properties observed at proactive caches (such as web proxies). Another reason for using $\mathrm{ZipF}$ distribution is its simplicity; 
TABLE 1: Simulation parameters.

\begin{tabular}{|c|c|c|}
\hline Parameter & Macrocell & Moving small-cell \\
\hline Cell layout & Hexagonal grid, 3 sectors per site & Uniform random distribution \\
\hline Radius of cell & $166 \mathrm{~m}(\mathrm{ISD}=3 R=500 \mathrm{~m})$ & $10 \mathrm{~m}$ \\
\hline Number of cells & 7 sites & $20 \sim 100$ \\
\hline \multicolumn{3}{|l|}{ Access link } \\
\hline Carrier freq. & $2 \mathrm{GHz}$ & $3.5 \mathrm{GHz}$ \\
\hline Bandwidth & $10 \mathrm{MHz}$ & $10 \mathrm{MHz}$ \\
\hline \multirow{2}{*}{ Tx power } & $46 \mathrm{dBm}$ (downlink) & $23 \mathrm{dBm}$ (downlink) \\
\hline & $23 \mathrm{dBm}$ (uplink) & $23 \mathrm{dBm}$ (uplink) \\
\hline \multicolumn{3}{|l|}{ Wireless $\mathrm{BH} / \mathrm{SH}$ link } \\
\hline Carrier freq. & - & $2 \mathrm{GHz}$ \\
\hline Bandwidth & - & $10 \mathrm{MHz}$ \\
\hline \multirow{2}{*}{ Tx power } & \multirow{2}{*}{-} & $46 \mathrm{dBm}$ (downlink of $\mathrm{BH}$ ) \\
\hline & & $23 \mathrm{dBm}$ (uplink of BH, SH) \\
\hline \multirow{2}{*}{ Antenna pattern } & \multirow{2}{*}{ Three-sector (2D) } & Three-sector (2D) (BH) \\
\hline & & Omnidirectional (2D) (SH) \\
\hline \multirow{2}{*}{ Antenna height } & MBS: $25 \mathrm{~m}$ & mSC: $2 \mathrm{~m}$ \\
\hline & MUE: $1.5 \mathrm{~m}$ & mSUE: $1.5 \mathrm{~m}$ \\
\hline Mobility model & MUE: random walk model & $\begin{array}{l}\text { mSC: random walk model } \\
\text { mSUE: group moving }\end{array}$ \\
\hline Sidehaul connection & - & $\begin{array}{l}\text { Contents and distance based } \\
\text { connection } \\
\text { (max distance: } 200 \mathrm{~m})\end{array}$ \\
\hline \multirow[t]{2}{*}{ Number of UE pieces per cell } & $300-(2 \cdot M)$ & \multirow[t]{2}{*}{2 per mSC } \\
\hline & $M=$ the number of mSCs & \\
\hline
\end{tabular}

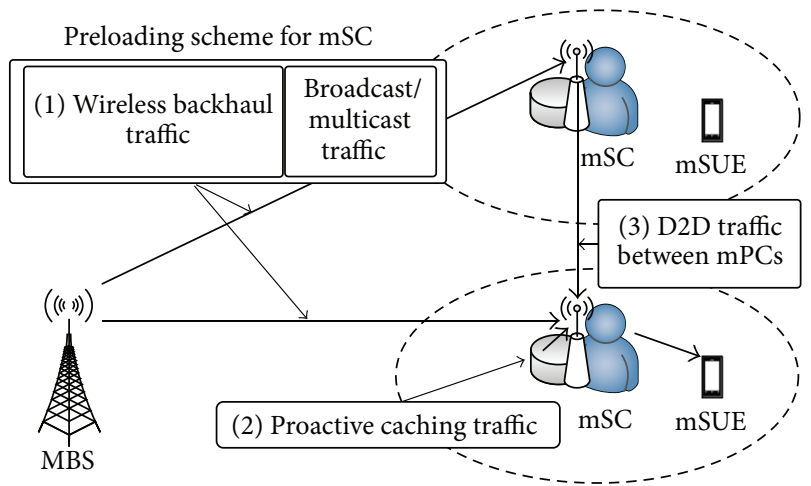

FIGURE 3: Off-peak time proactive caching scheme for mSC network.

we believe that the complexity cost of other machine learning algorithms will overburden the MSC network which have limited computational capabilities. In ZipF distribution, $\alpha$ is the characterization exponent that ranges from zero to one. Moreover, it is obvious that the performance of mSC network is decidedly dependent on cache size $(S)$. Huge cache size can significantly reduce the backhaul load and improve the QoS of mSC network. Furthermore, unlike broadcast mode, orthogonal radio resources are required for each multicast group transmission. Thus, the number of multicasting groups can significantly affect the performance of overall network.

\section{Performance Evaluation}

4.1. Simulation Environment. In order to evaluate the performance of our proposed mSC network with proactive cache, we conducted system level simulations. We consider a sevenmacrocell network, where each cell consists of three hexagonal sectors. MBSs are located in the center of each macrocell and the intercell distance is 500 meters. MUE and mSCs are randomly deployed and then they move within macrocells. Similarly, mSUE pieces are randomly and uniformly deployed and move within the coverage area of their serving mSCs. In order to capture the real time mobility pattern of mSCs, we have used random walk mobility model [35]. According to our considered random walk model the moving cell (which can be a public transportation vehicle) travels in a random direction with random velocity and flight time. More detailed simulation parameters are given in Table 1.

In our system level simulator, we have adopted ITU UMa and WINNER path loss models for macrocells and mSCs, respectively. ITU UMa model considers urban macrocell environment $[36,37]$. Pathloss equation of ITU UMa model is as follows:

$$
\begin{aligned}
\mathrm{PL} & =22.0 \log _{10}(d)+28.0+20 \log _{10}\left(f_{c}\right), \\
10 \mathrm{~m}<d<d_{\mathrm{BP}}, & \mathrm{PL}=40.0 \log _{10}(d)+7.8-18.0 \log _{10}\left(h_{\mathrm{BS}}\right)
\end{aligned}
$$




$$
\begin{aligned}
& -18.0 \log _{10}\left(h_{\mathrm{UT}}\right)+2.0 \log _{10}\left(f_{c}\right), \\
& d_{\mathrm{BP}}<d<5000 \mathrm{~m},
\end{aligned}
$$

where $d$ is distance between transmitter and receiver. $f_{c}$ is carrier frequency with range of 2 to $6 \mathrm{GHz}$. $h_{\mathrm{BS}}$ and $h_{\mathrm{UT}}$ are antenna heights of BS and UE, respectively, where $d_{\mathrm{BP}}$ is break point distance defined as

$$
d_{\mathrm{BP}}=\frac{4 h_{\mathrm{BS}} h_{\mathrm{UT}} f_{c}}{c}, \quad c=3.0 \cdot 10^{8} \mathrm{~m} / \mathrm{s} .
$$

WINNER model provides pathloss model for small-cells which has low power and small coverage area $[38,39]$ and its pathloss equations are

$$
\begin{aligned}
\mathrm{PL}_{B 1 \_ \text {total }}(d)= & \max \left(\mathrm{PL}_{\text {free }}(d), \mathrm{PL}_{B 1}(d)\right) \\
\mathrm{PL}_{\text {free }}(d)= & 20 \log _{10}(d)+46.4+20 \log _{10}\left(\frac{f_{c}}{2.0}\right) \\
\mathrm{PL}_{B 1}(d)= & \left(44.9-6.55 \log _{10}\left(h_{\mathrm{BS}}\right)\right) \log _{10}(d) \\
& +5.83 \log _{10}\left(h_{\mathrm{BS}}\right)+18.38 \\
& +23 \log _{10}\left(f_{c}\right),
\end{aligned}
$$

where $\mathrm{PL}_{\text {free }}(d)$ and $\mathrm{PL}_{B 1}(d)$ mean free space pathloss and pathloss for small-cell, respectively.

In this paper, we have used the overall network capacity $\left(C_{\text {Total }}\right)$ as a performance metric, which is total sum of macrocell capacity $\left(C_{\text {Macro }}\right)$ and $\mathrm{mSC}$ capacity $\left(C_{\mathrm{mSC}}\right)$ in downlink. The capacity of each cell depends on the spectral efficiency and bandwidth assigned to UE. Spectral efficiency of UE can be obtained as the relationship between the signal to interference and noise ratio (SINR) and modulation and coding scheme (MCS) table [36].

Let $U$ and $M$ denote the numbers of MUE pieces and mSCs deployed in each macrocell, respectively. $U_{k}$ denotes the number of mSUE pieces in the coverage of $\mathrm{mSC} k$. The total available bandwidths in $2 \mathrm{GHz}$ and $3.5 \mathrm{GHz}$ frequency bands are $W_{2 \mathrm{GHz}}$ and $W_{3.5 \mathrm{GHz}}$, respectively. We define the macrocell capacity $\left(C_{\text {Macro }}\right)$ as the sum of all MUE capacities. Thus, it can be calculated as

$$
C_{\text {Macro }}=(1-\rho) \frac{W_{2 \mathrm{GHz}}}{U+M} \sum_{i=1}^{U} \mathrm{MCS}_{\mathrm{DL}}\left(\operatorname{SINR}_{i}\right) \text {, }
$$

where $i$ means index of MUE attached to the MBS. $\rho(0 \leq$ $\rho \leq 1)$ depicts the ratio of radio resources for broadcasting/multicasting to overall radio resources for $2 \mathrm{GHz}$ downlink.

Likewise, the capacity of $\mathrm{mSC} k\left(C_{\mathrm{mSC}, k}\right)$ is also defined as the sum of all connected mSUE's capacities. However, the capacity of mSC depends on its transmission mode, that is, relay mode, cache mode, and $\mathrm{mSC}$-to-mSC (sidehaul) mode. If $\mathrm{mSUE}$ requests a content file not cached in its respective or neighboring $\mathrm{mSCs}$, the $\mathrm{mSC}$ performs relay transmission. In this case, the mSUE receives its data via wireless backhaul link and access link for mSUE. Thus, capacity of mSC $k\left(C_{\mathrm{mSC}, k}\right)$ in relay mode is defined as the minimum value between capacity

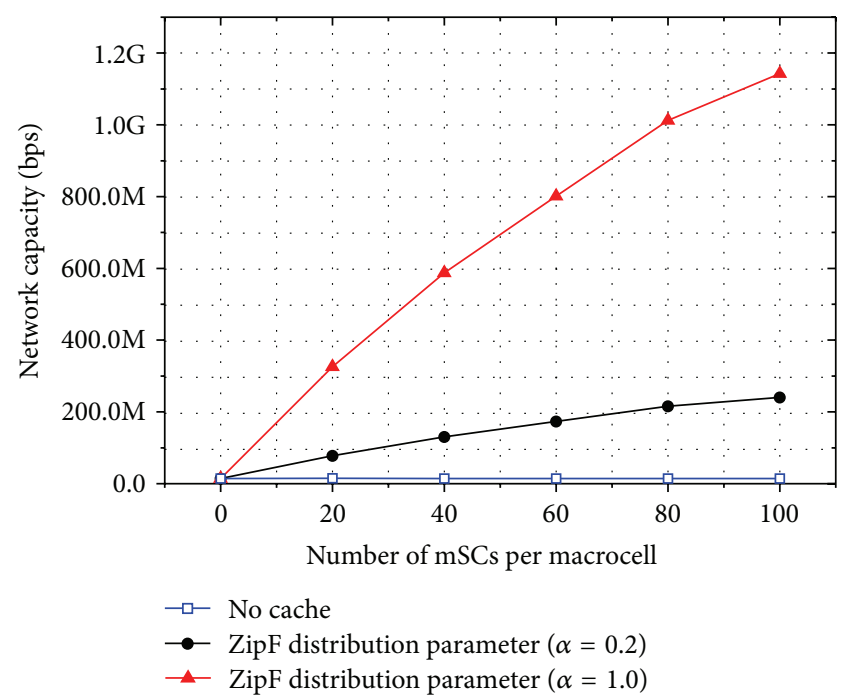

FIGURE 4: Network capacity varying numbers of mSCs operating in broadcast mode.

of wireless backhaul link $\left(C_{\mathrm{BH}, k}\right)$ and capacity of access link for $\mathrm{mSUE}\left(C_{\text {access }, k}\right)$ and it can be expressed as

$$
\begin{aligned}
C_{\mathrm{mSC}, k} & =\min \left(C_{\mathrm{BH}, k}, C_{\mathrm{access}, k}\right), \\
C_{\mathrm{BH}, k} & =(1-\rho) \frac{W_{2 \mathrm{GHz}}}{U+M} \operatorname{MCS}_{\mathrm{DL}}\left(\operatorname{SINR}_{\mathrm{BH}, k}\right), \\
C_{\text {access }, k} & =\frac{W_{3.5 \mathrm{GHz}}}{U_{k}} \sum_{j=1}^{U_{k}} \operatorname{MCS}_{\mathrm{DL}}\left(\operatorname{SINR}_{j}\right) .
\end{aligned}
$$

Similarly, if mSUE requests a content file that is available in the cache to its serving $\mathrm{mSC}$, the $\mathrm{mSC}$ performs cache transmission. In cache transmission mode, the mSUE directly receives its requested data from its serving $\mathrm{mSC}$ via access link. Thus, the capacity of mSC $k$ operating in cache mode can be determined by the capacity of its access link for mSUE $\left(C_{\text {access }, k}\right)$. On the other hand, mSCs operate in sidehaul transmission mode, if the contents requested by mSUE are not available in its serving $\mathrm{mSC}$ but are available in the cache of a neighboring $\mathrm{mSC}$. The neighboring $\mathrm{mSC}$ delivers such data to serving $\mathrm{mSC}$ via wireless sidehaul link. The serving $\mathrm{mSC}$ forwards the received data to its respective mSUE via access link. In this case, capacity of $\operatorname{mSC} k\left(C_{\mathrm{mSC}, k}\right)$ is decided as the minimum value between capacity of wireless sidehaul link $\left(C_{\mathrm{SH}, k}\right)$ and access link $\left(C_{\mathrm{access}, k}\right)$, and it can be expressed as

$$
\begin{aligned}
& C_{\mathrm{mSC}, k}=\min \left(\delta \cdot C_{\mathrm{SH}, k}, C_{\text {access }, k}\right) \text {, } \\
& \delta= \begin{cases}0, & \text { if sidehaul link does not exist } \\
1, & \text { if sidehaul link exists, }\end{cases} \\
& C_{\mathrm{SH}, k}=W_{2 \mathrm{GHz}} \mathrm{MCS}_{\mathrm{UL}}\left(\operatorname{SINR}_{\mathrm{SH}, k}\right) \text {. }
\end{aligned}
$$

4.2. Simulation Results. Figure 4 shows the overall network capacity with varying number of mSCs operating in broadcast mode. It depicts that the overall network capacity is 


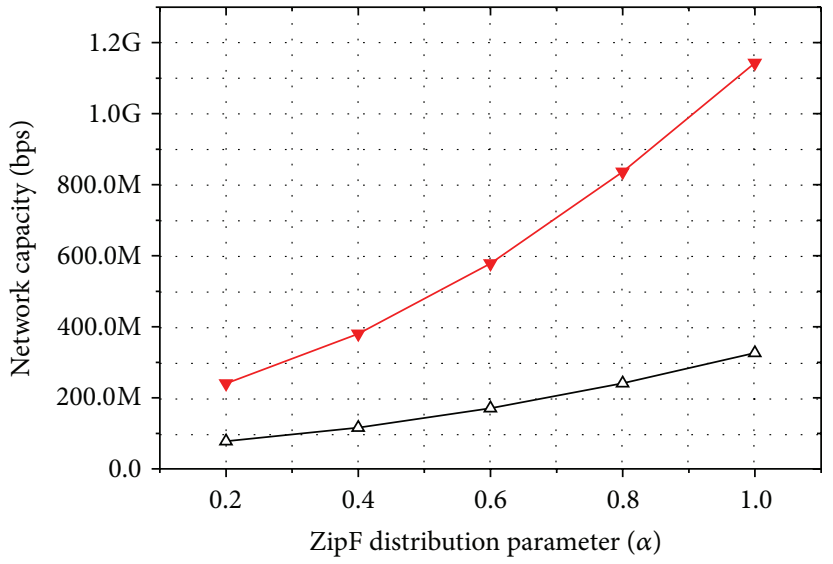

$M$ (number of mPCs)

$\rightarrow-20$

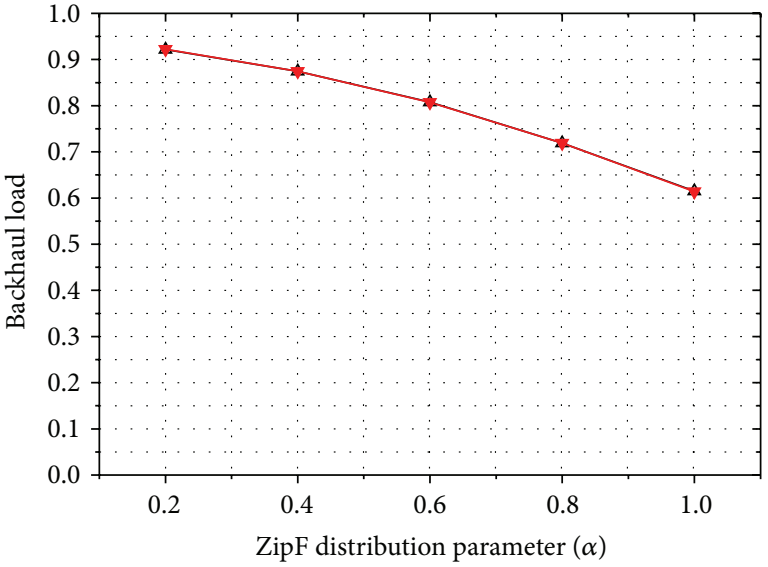

$M$ (number of mPCs)

$-\triangle-20$

$\rightarrow 100$

(a) Network capacity

(b) Backhaul load

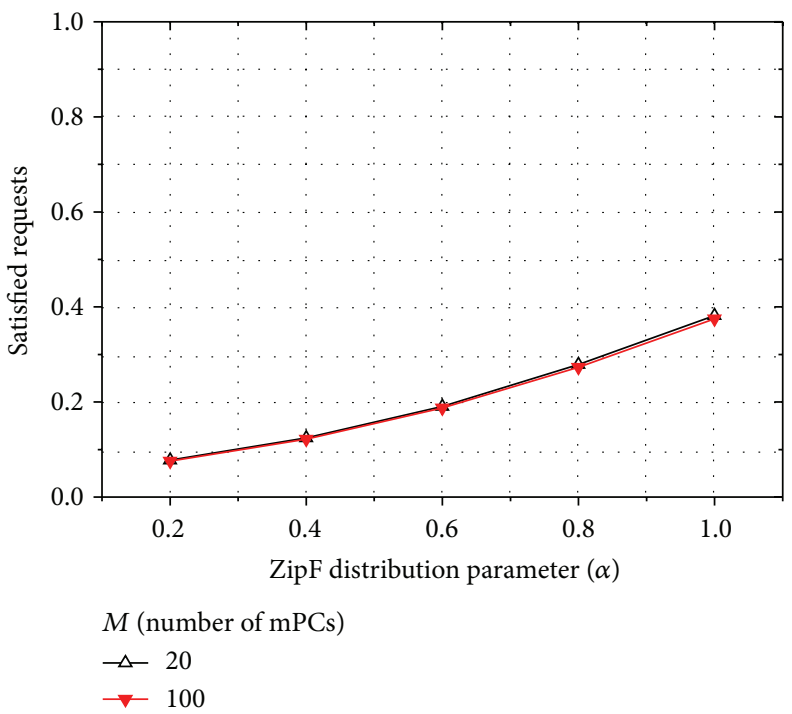

(c) Number of satisfied requests

FIGURE 5: Effect of ZipF distribution $(\alpha)$ on overall network performance operating at broadcasting and multicasting mode.

highly dependent on number of mSCs in the cell. It also shows that the mSC with cache scenario outperforms the no-cache scenario, because most of the contents requested by mSUE are already available in the cache of mSCs. Furthermore, as the popularity of files increases ( $\alpha$ increases.) the overall network capacity also increases. It is because more mSUE pieces request the already cached files.

Similarly, Figure 5 depicts the effect of ZipF distribution $(\alpha)$ on overall network capacity, backhaul load, and number of satisfied requests. Two different $\mathrm{mSC}$ deployment scenarios (sparse and dense) are considered. Figure 5(a) shows that, beside the inter-mSC interference, the overall network capacity in dense deployment scenario $(100 \mathrm{mSCs}$ per macrocell) is significantly higher than sparse deployment scenario ( $20 \mathrm{mSCs}$ per macrocell). The reason is that each $\mathrm{mSC}$ uses the same $2 \mathrm{GHz}$ frequency band in access link. In dense deployment, more mSCs reuse the same frequency band in their access links. Likewise, Figure 5(b) depicts that the backhaul load significantly reduces as the file popularity increases. Furthermore, it also shows that, in both deployment scenarios, the file popularity has no major effect on backhaul load. In this work, we define backhaul load as the ratio of number of mSCs using backhaul link over total number of mSCs. Similarly, Figure 5(c) illustrates the relation between satisfied requests and file popularity. It shows that in both deployment scenarios the number of satisfied requests increases as the popularity of file increases. Here the term of satisfied requests means the ratio between numbers of 


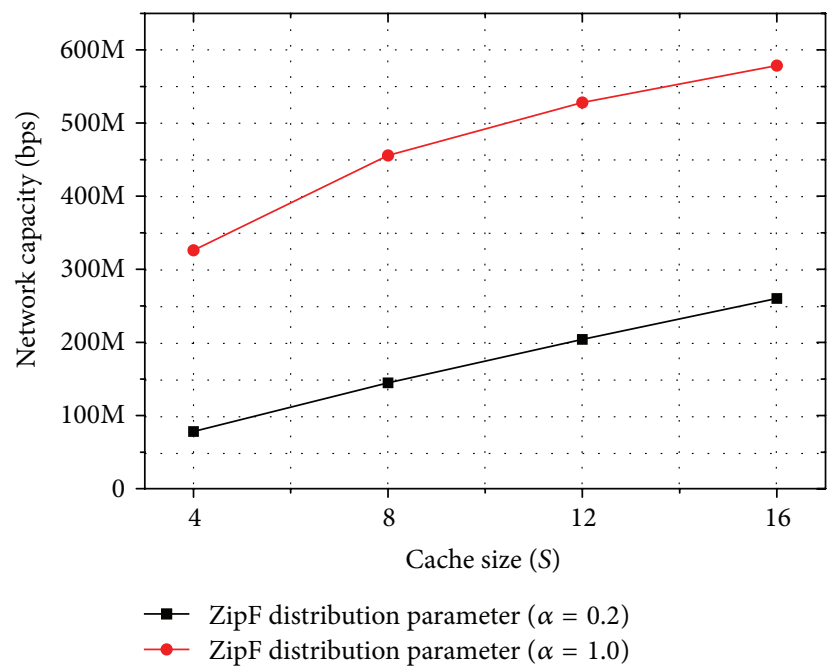

(a) Network capacity

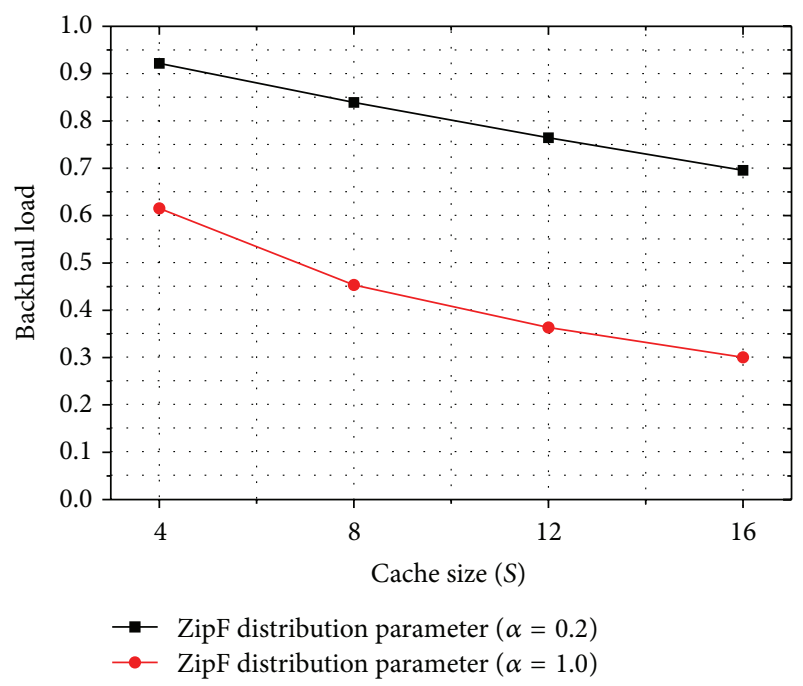

(b) Backhaul load

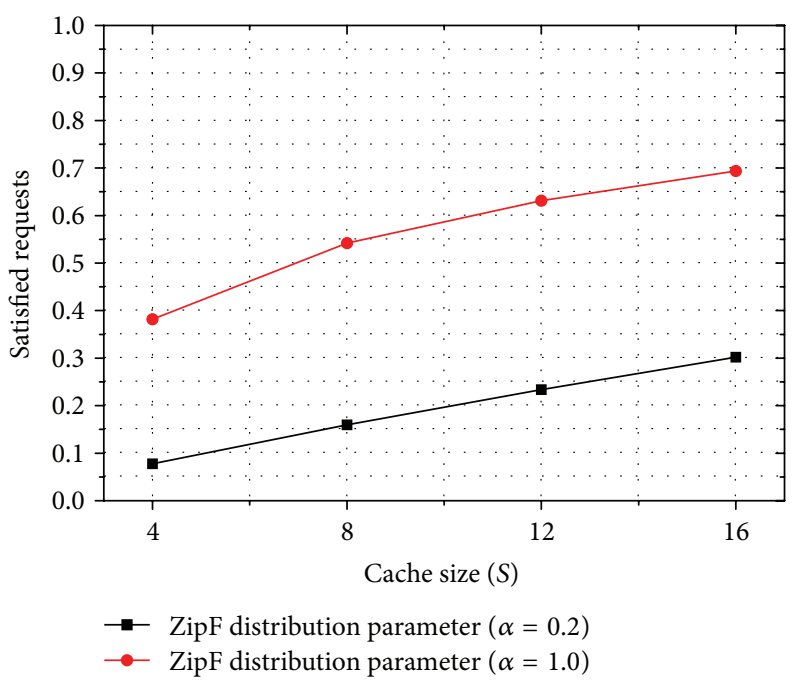

(c) Number of satisfied requests

FIGURE 6: Effect of cache size $(S)$ on overall network performance with varying ZipF distribution parameters.

satisfied requests over total number of requests. If a user successfully receives a file size of $1 \mathrm{MB}$ within 1 second after his request, we call this request as satisfied one.

The effect of cache size $(S)$ on overall network capacity, backhaul load, and number of satisfied requests is shown in Figure 6. It is shown in Figure 6(a) that, with fixed number of mSCs (in this case 20), the overall network capacity significantly increases as the cache size increases. Since large cache size can proactively store popular contents, they can also significantly reduce the backhaul traffic load (Figure 6(b)) and increase the numbers of satisfied user requests (Figure 6(c)) in mSC network.

Figure 7 shows the effect of multicast groups on overall network capacity. It can be observed from Figures 7(a) and 7 (b) that, for two different zip distribution parameters $(\alpha=$ 0.2 and $\alpha=1$ ), the broadcast mode outperforms the multicast mode. It is because the MBS in multicast mode uses orthogonal channels to transmit different contents to different $\mathrm{mSC}$ groups (in this case 2 groups), and thus it consumes more backhaul bandwidth than broadcast mode. Figure 7(c) depicts the comparison of different resource utilization of mSCs operating at broadcast and multicast modes. It can be observed that in both broadcast and multicast mode the ZipF distribution factor plays a vital role and the backhaul load reduces to $61 \%$ and $59 \%$ when it approaches to 1 , respectively. Furthermore, the utilization of sidehaul link in multicast mode increases up to $14 \%$ when $\alpha$ approaches to 1 .

\section{Conclusion}

In this paper, we discuss the role of mSCs in future HetNets and proposed a novel proactive caching based mSC network. We show that, by using the predictive nature of user demands, next generation networks can effectively preload their cache 


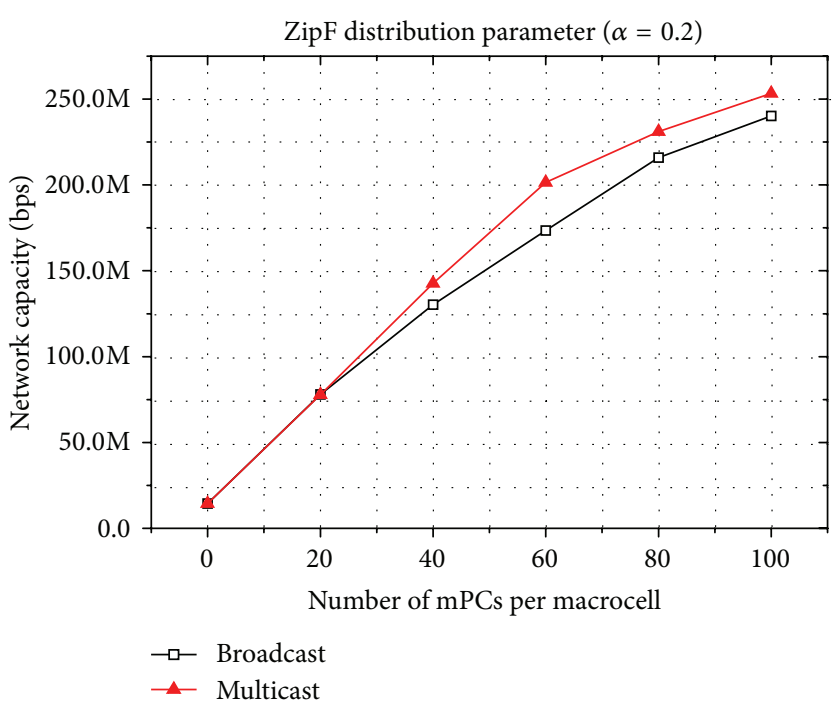

(a) Network capacity when $\alpha=0.2$

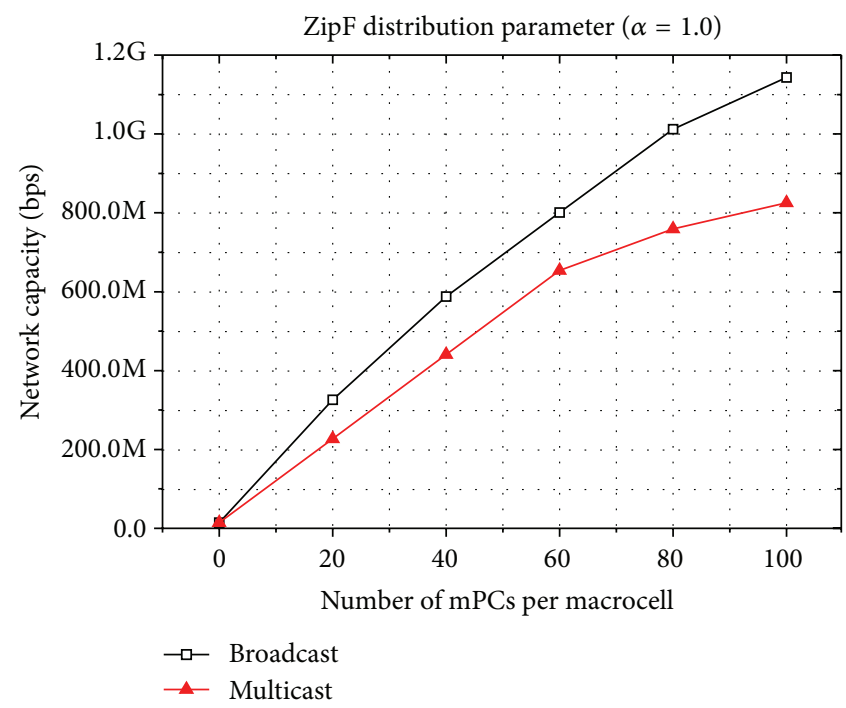

(b) Network capacity when $\alpha=1.0$

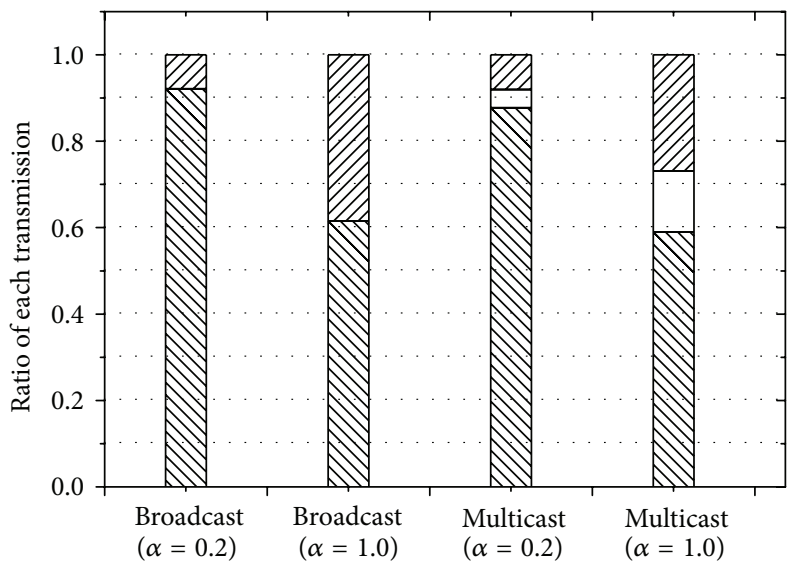

$\triangle 1$ Backhaul transmission

VIJ Caching transmission

D2D transmission

(c) Ratio of different transmission modes

FIGURE 7: Influence of multicast groups on overall network performance with varying values of $\alpha$.

with popular contents and reduce the traffic data demand in peak hours. Our extensive system level simulation results show that the proposed mSC network can significantly improve the QoS performance and overall system capacity of the network. We also show that the overall network performance is highly dependent on number of mSCs deployed, cache size, and content popularity. For future studies, we are aiming at incorporating the transmitted power control schemes in our simulator, which will effectively mitigate cross- and cotier interference in mSC networks. Another interesting line of investigation is to study various resource partitioning and scheduling schemes, which can statically or dynamically divide radio resources between macrocell and mSCs and reduce the interference and improve overall performance of the network.

\section{Competing Interests}

The authors declare that they have no competing interests.

\section{Acknowledgments}

This work was supported by Institute for Information \& communications Technology Promotion (IITP) grant funded by the Korean government (MSIP) (no. R0101-15-244; Development of 5G Mobile Communication Technologies for Hyperconnected Smart Services).

\section{References}

[1] Cisco, "Global mobile data traffic forecast update, 2013-2018," White Paper, 2014. 
[2] A. Damnjanovic, J. Montojo, Y. Wei et al., "A survey on 3GPP heterogeneous networks," IEEE Wireless Communications, vol. 18, no. 3, pp. 10-21, 2011.

[3] S.-P. Yeh, S. Talwar, G. Wu, N. Himayat, and K. Johnsson, "Capacity and coverage enhancement in heterogeneous networks," IEEE Wireless Communications, vol. 18, no. 3, pp. 32-38, 2011.

[4] D. López-Pérez, A. Valcarce, G. De La Roche, and J. Zhang, "OFDMA femtocells: a roadmap on interference avoidance," IEEE Communications Magazine, vol. 47, no. 9, pp. 41-48, 2009.

[5] X. Ge, H. Cheng, M. Guizani, and T. Han, "5G wireless backhaul networks: challenges and research advances," IEEE Network, vol. 28, no. 6, pp. 6-11, 2014.

[6] T. Yamamoto and S. Konishi, "Impact of small cell deployments on mobility performance in LTE-advanced systems," in Proceedings of the IEEE 24th International Symposium on Personal, Indoor and Mobile Radio Communications (PIMRC Workshops '13), pp. 189-193, London, UK, September 2013.

[7] 3GPP, "Evolved Universal Terrestrial Radio Access (E-UTRA) and Evolved Universal Terrestrial Radio Access Network (EUTRAN); Overall description; Stage 2," 3rd Generation Partnership Project (3GPP), TS 36.300, v12.3.0, March 2011, http:// www.3gpp.org/ftp/Specs/html-info/36300.htm.

[8] Y. Sui, J. Vihriala, A. Papadogiannis, M. Sternad, W. Yang, and T. Svensson, "Moving cells: a promising solution to boost performance for vehicular users," IEEE Communications Magazine, vol. 51, no. 6, pp. 62-68, 2013.

[9] L. Chen, Y. Huang, F. Xie et al., "Mobile relay in LTE-advanced systems," IEEE Communications Magazine, vol. 51, no. 11, pp. 144-151, 2013.

[10] 3GPP, "Evolved Universal Terrestrial Radio Access (E-UTRA); Study on mobile relay," 3rd Generation Partnership Project (3GPP), TR 36.836, v2.0.2, June 2014, http://www.3gpp.org/ dynareport/36836.htm.

[11] Y. Sui, Z. Ren, W. Sun, T. Svensson, and P. Fertl, "Performance study of fixed and moving relays for vehicular users with multicell handover under co-channel interference," in Proceedings of the 2nd IEEE International Conference on Connected Vehicles and Expo (ICCVE '13), pp. 514-520, IEEE, Las Vegas, Nev, USA, December 2013.

[12] A. Enrique, "User-centric wireless local loop use-cases: a brief overview on assumptions and requirements," White Paper, 2011.

[13] A. Ghosh, N. Mangalvedhe, R. Ratasuk et al., "Heterogeneous cellular networks: from theory to practice," IEEE Communications Magazine, vol. 50, no. 6, pp. 54-64, 2012.

[14] E. Baştug, J.-L. Guénégo, and M. Debbah, "Proactive small cell networks," in Proceedings of the 20th International Conference on Telecommunications (ICT '13), pp. 1-5, Casablanca, Morocco, May 2013.

[15] L. Chen, W. Chen, B. Wang, X. Zhang, H. Chen, and D. Yang, "System-level simulation methodology and platform for mobile cellular systems," IEEE Communications Magazine, vol. 49, no. 7, pp. 148-155, 2011.

[16] Y. M. Kwon, J. Shin, J. S. Kim, S.-M. Oh, M. Y. Chung, and A.S. Park, "Development of system level simulator for evaluating performance of moving personalcell network," in Proceedings of the 8th ACM International Conference on Ubiquitous Information Management and Communication (ICUIMC '14), p. 19, Siem Reap, Cambodia, January 2014.

[17] I. Hwang, B. Song, and S. S. Soliman, "A holistic view on hyper-dense heterogeneous and small cell networks," IEEE Communications Magazine, vol. 51, no. 6, pp. 20-27, 2013.
[18] J. G. Andrews, S. Buzzi, W. Choi et al., "What will 5G be?" IEEE Journal on Selected Areas in Communications, vol. 32, no. 6, pp. 1065-1082, 2014.

[19] H. S. Dhillon, R. K. Ganti, F. Baccelli, and J. G. Andrews, "Modeling and analysis of K-tier downlink heterogeneous cellular networks," IEEE Journal on Selected Areas in Communications, vol. 30, no. 3, pp. 550-560, 2012.

[20] V. Van Phan, K. Horneman, L. Yu, and J. Vihriala, "Providing enhanced cellular coverage in public transportation with smart relay systems," in Proceedings of the IEEE Vehicular Networking Conference (VNC '10), pp. 301-308, Jersey City, NJ, USA, December 2010.

[21] Y. Sui, A. Papadogiannis, W. Yang, and T. Svensson, "Performance comparison of fixed and moving relays under cochannel interference," in Proceedings of the IEEE Globecom Workshops (GC Wkshps '12), pp. 574-579, IEEE, Anaheim, Calif, USA, December 2012.

[22] Y. Sui, A. Papadogiannis, and T. Svensson, "The potential of moving relays-a performance analysis," in Proceedings of the IEEE 75th Vehicular Technology Conference (VTC Spring '12), pp. 1-5, IEEE, Yokohama, Japan, May 2012.

[23] Y. Sui, A. Papadogiannis, W. Yang, and T. Svensson, "The energy efficiency potential of moving and fixed relays for vehicular users," in Proceedings of the IEEE 78th Vehicular Technology Conference (VTC Fall '13), pp. 1-7, IEEE, Las Vegas, Nev, USA, September 2013.

[24] IEEE 802.16 Broadband Wireless Access Working Group, "IEEE C802.16j-07/087r2 mobile relay station operation," IEEE, Technical Report, 2007, http://www.ieee802.org/16/.

[25] J. Tadrous, A. Eryilmaz, and H. El Gamal, "Proactive content download and user demand shaping for data networks," IEEE/ACM Transactions on Networking, vol. 23, no. 6, pp. 19171930, 2013.

[26] N. Golrezaei, K. Shanmugam, A. G. Dimakis, A. F. Molisch, and G. Caire, "Femtocaching: wireless video content delivery through distributed caching helpers," in Proceedings of the IEEE (INFOCOM '12), pp. 1107-1115, IEEE, Orlando, Fla, USA, 2012.

[27] M. Ji, G. Caire, and A. F. Molisch, "Fundamental limits of distributed caching in D2D wireless networks," in Proceedings of the IEEE Information Theory Workshop (ITW '13), pp. 1-5, IEEE, Sevilla, Spain, September 2013.

[28] E. Bastug, M. Bennis, and M. Debbah, "Living on the edge: the role of proactive caching in $5 \mathrm{G}$ wireless networks," IEEE Communications Magazine, vol. 52, no. 8, pp. 82-89, 2014.

[29] A. Bar-Noy, R. E. Ladner, and T. Tamir, "Optimal delay for media-on-demand with pre-loading and pre-buffering," Theoretical Computer Science, vol. 399, no. 1-2, pp. 3-11, 2008.

[30] J. Lee, M. Sun, and G. Lebanon, "A comparative study of collaborative filtering algorithms," https://arxiv.org/abs/1205.3193.

[31] R. Vale and H. Viswanathan, "Further Efficiencies with eMBMS Preloading," https://techzine.alcatel-lucent.com/further-efficiencies-embms-preloading.

[32] G. K. Zipf, "Relative frequency as a determinant of phonetic change," Harvard Studies in Classical Philology, vol. 40, pp. 1-95, 1929.

[33] K. Shanmugam, N. Golrezaei, A. G. Dimakis, A. F. Molisch, and G. Caire, "FemtoCaching: wireless content delivery through distributed caching helpers," IEEE Transactions on Information Theory, vol. 59, no. 12, pp. 8402-8413, 2013.

[34] L. Breslau, P. Cao, L. Fan, G. Phillips, and S. Shenker, "Web caching and Zipf-like distributions: evidence and implications," 
in Proceedings of the IEEE 18th Annual Joint Conference of the IEEE Computer and Communications Societies (INFOCOM '99), vol. 1, pp. 126-134, New York, NY, USA, March 1999.

[35] D. B. Johnson and D. A. Maltz, "Dynamic source routing in ad hoc wireless networks," in Mobile Computing, T. Imielinski and H. Korth, Eds., chapter 5, pp. 153-181, Kluwer Academic, Dordrecht, Netherlands, 1996.

[36] 3GPP, "Further advancements for E-UTRA physical layer aspects (Release 9)," 3rd Generation Partnership Project (3GPP) TR 36.814, v9.0.0, 2010.

[37] 3GPP, "Evolved Universal Terrestrial Radio Access (E-UTRA); Radio Frequency (RF) system scenarios (Release 11)," 3rd Generation Partnership Project (3GPP), TR 36.942, v1l.0.0, September 2012.

[38] IST-4-027756 WINNER II D1.1.2V1.2, WINNER II Channel Models, 2007.

[39] D5.3: WINNER+ Final Channel Models, V1.0, 2010. 

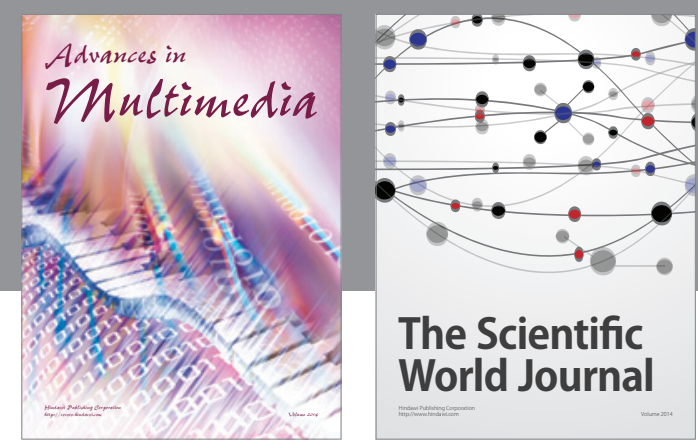

The Scientific World Journal
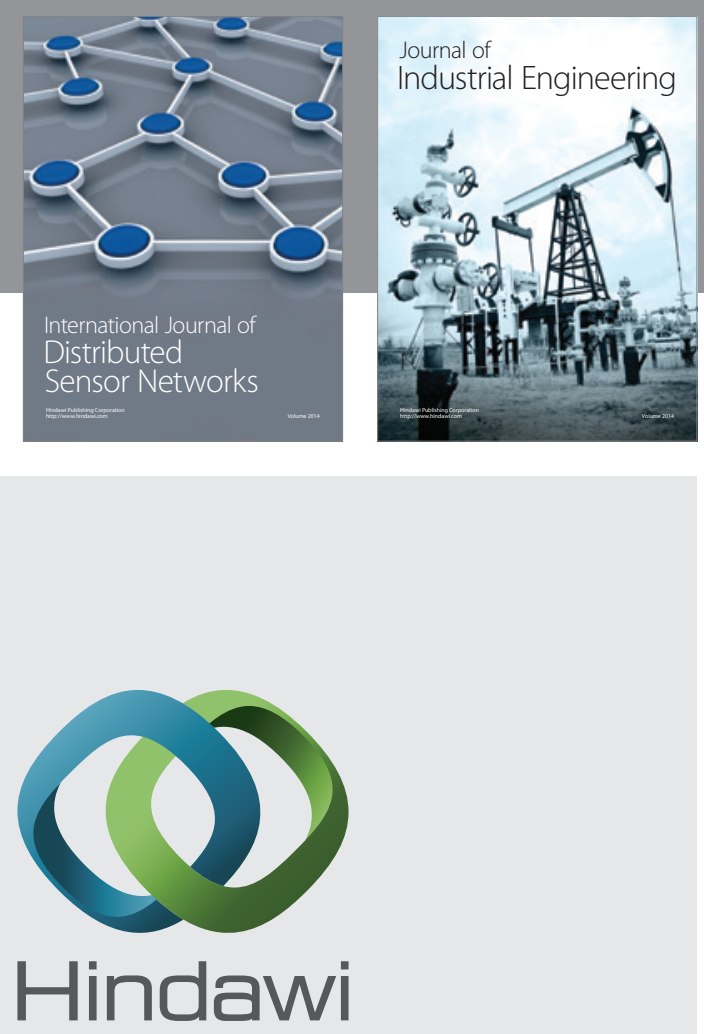

Submit your manuscripts at

http://www.hindawi.com

\section{Computer Networks} and Communications
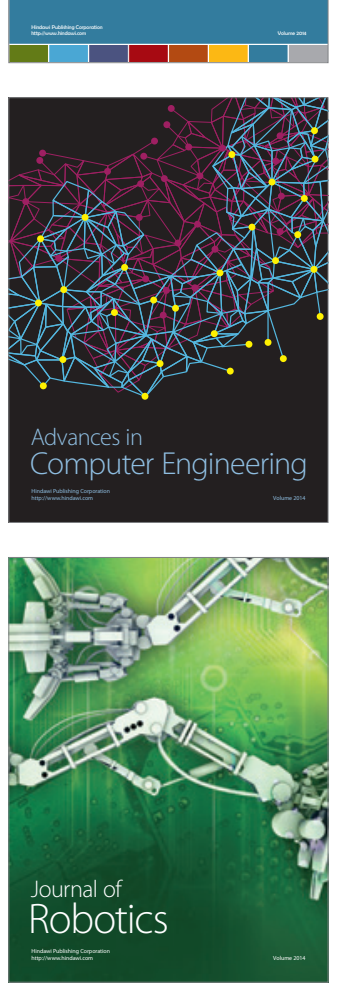
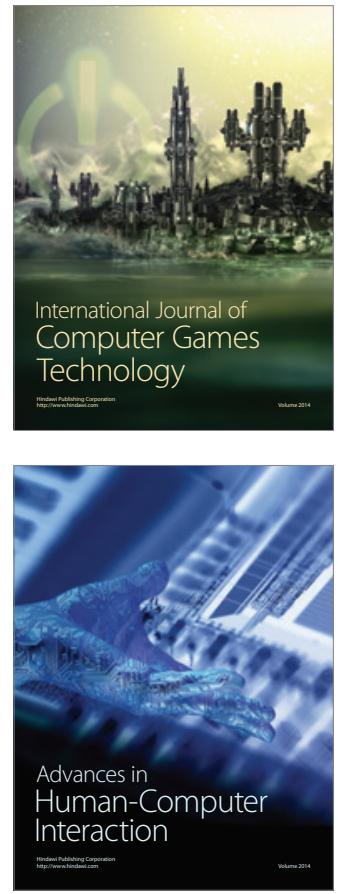
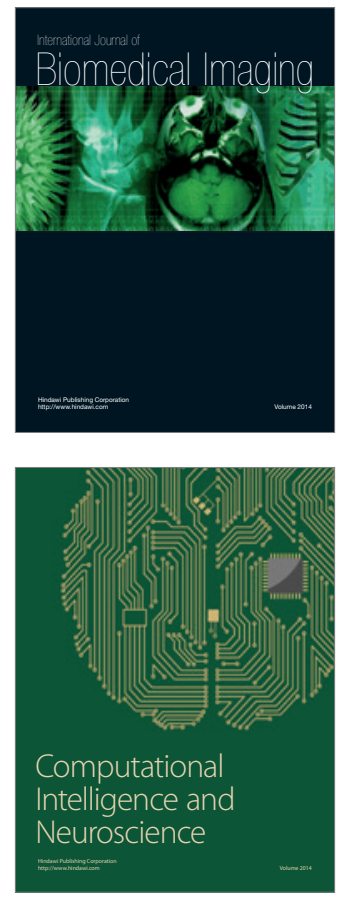
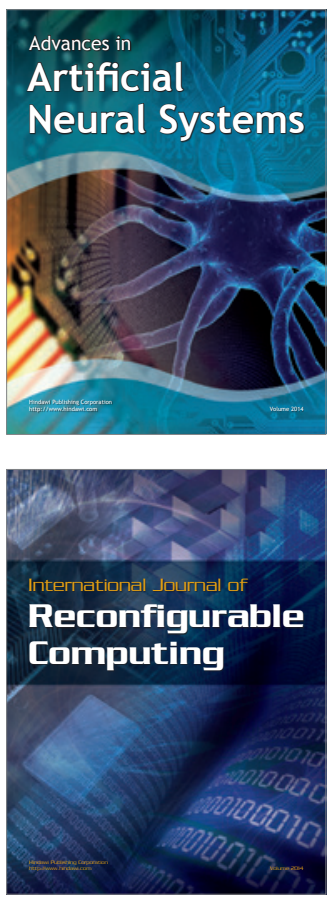
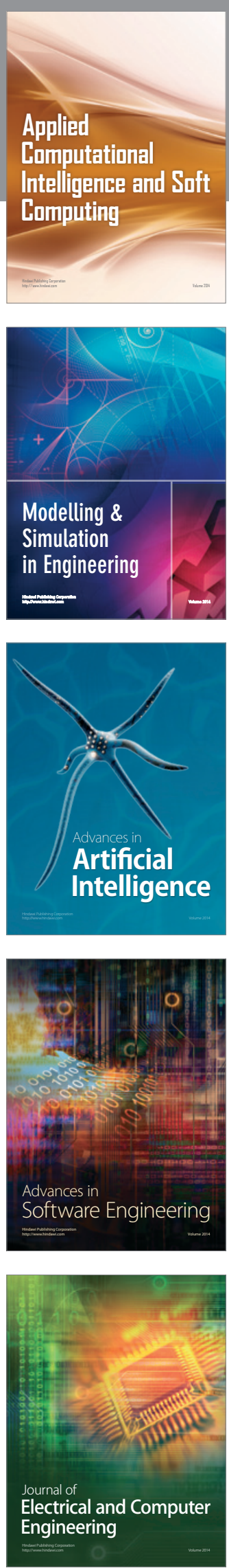